Silvia Scoccianti, MD

Radioterapia, Università di Firenze

Azienda Ospedaliera Universitaria Caregg

Firenze, Italy

Stefano Maria Magrini, MD

stituto del Radio "O. Alberti",

Dipartimento di Radioterapia Oncologica,

Università di Brescia, Brescia, Italy

Umberto Ricardi, MD

Radioterapia Oncologica, Universita' di Torino,

Azienda Ospedaliera Universitaria San Giovann

Battista, Torino, Italy

Beatrice Detti, MD

Radioterapia, Università di Firenze

Azienda Ospedaliera Universitaria Caregg

Firenze, Italy

Michela Buglione, MD

Istituto del Radio "O. Alberti",

Dipartimento di Radioterapia Oncologica,

Università di Brescia, Brescia, Italy

Guido Sotti, MD

Istituto Oncologico Veneto IRCCS,

Padova, Italy

Marco Krengli, MD

Radioterapia, Università del Piemonte Orientale,

Novara, Italy

Sergio Maluta, MD

Radioterapia Oncologica

Azienda Ospedaliera di Verona, Verona, Italy

Salvatore Parisi, MD

Radioterapia, IRCCS Ospedale

"Casa Sollievo della Sofferenza",

San Giovanni Rotondo, Italy

Filippo Bertoni, MD

Radioterapia Oncologica AOU Policlinico di

Modena, Modena, Italy

Cristina Mantovani, MD

Radioterapia Oncologica, Universita' di Torino,

Azienda Ospedaliera Universitaria San Giovann

Battista, Torino, Italy

Vincenzo Tombolini, MD

Cattedra di Radioterapia,

Facoltà Medicina e Chirurgia

Università de L'Aquila, L'Aquila, Italy

Costantino De Renzis, MD

Radioterapia Oncologica,

Dipartimento di Scienze Radiologiche,

AOU Policlinico G. Martino, Messina, Italy

Marco Lioce, MD

Radioterapia Oncologica

A.O.R.N. "G. Rummo", Benevento, Italy

Lucia Fatigante, MD

Radioterapia Oncologica, AOU Pisana, Pisa, Italy

Vincenzo Fusco, MD

Radioterapia, CROB-IRCCS, Rionero in Vulture, Italy

Paolo Muto, MD

Radioterapia, P.O. Ascalesi, Naples, Italy

Franco Berti, MD

Istituto Oncologico Veneto IRCCS, Padova, Italy

Giovanni Rubino, MD

Radioterapia Oncologica,

Dipartimento di Patologia umana e Oncologia,

Università di Siena, Siena, Italy

Samantha Cipressi, MD

Radioterapia, Università di Firenze,

Azienda Ospedaliera Universitaria Careggi,

Firenze, Italy

Laura Fariselli, MD

Radioterapia, Fondazione Istituto Neurologico

Carlo Besta, Milan, Italy

Marco Lupattelli, MD

Radioterapia Oncologica, Università di Perugia,

Ospedale Santa Maria Misericordia, Perugia, Italy

Riccardo Santoni, MD

Radioterapia, Università Tor Vergata

Rome, Italy

Luigi Pirtoli, MD

Radioterapia Oncologica,

Dipartimento di Patologia umana e Oncologia,

Università di Siena,

Siena, Italy

\title{
Patterns of Care and Survival in a Retrospective Analysis of 1059 Patients With Glioblastoma Multiforme Treated Between 2002 and 2007: A Multicenter Study by the Central Nervous System Study Group of Airo (Italian Association of Radiation Oncology)
}

OBJECTIVE: To investigate the pattern of care and outcomes for newly diagnosed glioblastoma in Italy and compare our results with the previous Italian Patterns of Care study to determine whether significant changes occurred in clinical practice during the past 10 years. METHODS: Clinical, pathological, therapeutic, and survival data regarding 1059 patients treated in 18 radiotherapy centers between 2002 and 2007 were collected and retrospectively reviewed.

RESULTS: Most patients underwent both computed tomography and magnetic resonance imaging either preoperatively (62.7\%) or postoperatively (35.5\%). Only 123 patients (11.6\%) underwent a biopsy. Radiochemotherapy with temozolomide was the most frequent adjuvant treatment $(70.7 \%)$. Most patients $(88.2 \%)$ received 3-dimensional conformal radiotherapy. Median survival was 9.5 months. Two- and 5 -year survival rates were $24.8 \%$ and $3.9 \%$, respectively. Multivariate analysis showed the statistical significance of age, postoperative Karnofsky Performance Status scale score, surgical extent, use of 3-dimensional conformal radiotherapy, and use of chemotherapy. Use of a more aggressive approach was associated with longer survival in elderly patients. Comparing our results with those of the subgroup of patients included in our previous study who were treated between 1997 and 2001, relevant differences were found: more frequent use of magnetic resonance imaging, surgical removal more common than biopsy, and widespread use of 3-dimensional conformal radiotherapy + temozolomide. Furthermore, a significant improvement in terms of survival was noted $(P<.001)$.

CONCLUSION: Changes in the care of glioblastoma over the past few years are documented. Prognosis of glioblastoma patients has slightly but significantly improved with a small but noteworthy number of relatively long-term survivors.

KEY WORDS: Chemotherapy, Database, Glioblastoma, Patterns of care, Radiotherapy

Neurosurgery 67:446-458, 2010

DOI: 10.1227/01.NEU.0000371990.86656.E8

www.neurosurgery-online.com

$\mathrm{T}$ he goal of the Central Nervous System Study Group of AIRO (Italian Association of Radiation Oncology), founded in 2005,

\begin{tabular}{ll}
\hline Giampaolo Biti, MD & $\begin{array}{l}\text { Azienda Ospedaliera Universitaria } \\
\text { Radioterapia, }\end{array}$ \\
Careggi, \\
Università di Firenze, & Viale Morgagni 85, \\
Azienda Ospedaliera Universitaria & 50134 Florence, Italy. \\
Careggi, & E-mail: silvia.scoccianti@unifi.it \\
Firenze, Italy & Received, June 22, 2009. \\
Reprint requests: & Accepted, May 1, 2010. \\
Silvia Scoccianti, MD, & Copyright $\odot 2010$ by the \\
Radioterapia, & Congress of Neurological Surgeons \\
Università di Firenze, &
\end{tabular}

is to promote clinical research on the management of brain tumors and to develop educa-

ABBREVIATIONS: 3D-CRT, 3-dimensional conformal radiotherapy; EORTC/NCIC, European Organization for Research and Treatment of Cancer and the National Cancer Institute of Canada; GBM glioblastoma multiforme; GOP, Glioma Outcomes Project; KPS, Karnofsky Performance Status; RPA, recursive partitioning analysis; $\mathbf{R T}$, radiotherapy; TMZ, temozolomide 
TABLE 1. Radiation Therapy Oncology Group Acute Radiation Morbidity Scoring Criteria for the Central Nervous System

\begin{tabular}{lllll}
\hline Grade $\mathbf{0}$ & \multicolumn{1}{c}{ Grade 1 } & \multicolumn{1}{c}{ Grade 2 } & \multicolumn{1}{c}{ Grade 3 } & \multicolumn{1}{c}{ Grade 4 } \\
\hline None & Fully functional status (ie, able & Neurological findings present & Neurological findings & Serious neurological impairment \\
& to work) with minor & sufficient to require home care; & requiring hospitalization for & that includes paralysis, coma, and \\
& neurological findings; no & nursing assistance may be & initial management & seizures $>3$ per week despite \\
& medication needed & required; medications including & & medication; hospitalization \\
& & steroids and antiseizure agents & & required \\
& & &
\end{tabular}

tional programs for improving the care of neuro-oncological patients.

The group collected clinical data on patients with high-grade gliomas treated in radiation oncology centers to perform a patterns of care study, evaluating the national standards of practice during the most recent years. The clinical presentations, surgical management, and adjuvant therapies for 1374 patients with highgrade gliomas including glioblastoma multiforme (GBM) and anaplastic astrocytoma treated in Italy between 2002 and 2007 were recorded using a common database. The current study focuses on patients with newly diagnosed GBM.

For many years, surgery and adjuvant radiotherapy (RT) have been the mainstay of therapy for GBM. After publication of the results of several clinical studies and, more recently, of a large randomized multicenter phase III trial performed by EORTC/NCIC (European Organization for Research and Treatment of Cancer and the National Cancer Institute of Canada), ${ }^{1}$ concomitant and sequential temozolomide (TMZ) became the standard of care for patients with GBM. The median survival of patients with GBM was 14.6 months in the recently published updated results of that trial. ${ }^{2}$

In this study, we investigated whether patterns of practice in Italy have changed in the recent past.

We previously reported the results of the Patterns of Care study of 1722 adult astrocytoma patients (GBM, $n=1235$; anaplastic astrocytoma, $\mathrm{n}=309$; low-grade gliomas, $\mathrm{n}=178$ ) treated between 1985 and $2001 .^{3}$

In the current study, a second national survey was done to compare current treatment modalities and outcomes of glioblastoma patients with data from the earlier Italian Patterns of Care study. In this retrospective database-driven study of $1059 \mathrm{GBM}$ patients treated in 18 RT centers between 2002 and 2007 we intended to provide a comparison with the previous Italian Patterns of Care study, calling attention to the changes in treatment of glioblastoma over the time period in which data on TMZ efficacy were widely disseminated.

\section{METHODS AND MATERIALS}

The Central Nervous System Study Group of AIRO designed a database to collect clinical data on patients with GBM. Several meetings were held with the participating centers to ensure the homogeneity of data collection. The coordinating study center (University of Florence) created and managed the central computerized database. Each participating center sent data forms to the central coordinating center. Queries could be e-mailed from participants to the registry staff for assistance and resolution of the problems. Moreover, periodic meetings with the investigators were arranged to offer assistance in completing the forms. Audits were performed to find omissions or inconsistencies, and registry staff contacted each center to resolve any ambiguities in responses. Only completed and checked data were stored in the central database.

The study was approved by the institutional review board of the coordinating center and of each participating institution. All enrolled patients gave informed consent.

A total of 1059 glioblastoma patients, treated between January 2002 and June 2007 in 18 Italian RT centers, were collected. The contribution of each center to the entire data set ranged from $0.3 \%$ to $14.3 \%$.

Detailed data regarding clinical presentation, surgical procedure, pathological specimen, postoperative RT, adjuvant chemotherapy, treatmentrelated morbidity, and survival outcome were recorded in the central database.

Only biopsy-proven pure glioblastoma cases, analyzed by specialist neuropathologists, were included in the study, excluding mixed gliomas with an oligodendroglial component.

Toxicity during adjuvant RT and/or chemotherapy was scored with Common Terminology Criteria for Adverse Events v3.0 (CTCAE) [http://ctep.cancer.gov/forms/CTCAEv3.pdf]. Worsening of neurological symptoms during RT was scored according to Central Nervous System Acute Radiation Morbidity Scoring Criteria [www.rtog.org/members/ toxicity/acute.html], (Table 1).

\section{Statistical Analysis}

The Kaplan-Meier method was used to estimate overall survival (OS). OS was calculated from the date of surgery until the time of death or last follow-up examination. Survival curves were compared using the logrank test. Multivariate analysis was performed with the Cox regression model. Differences between different subgroups of patients were calculated using the $\chi^{2}$ test. All analyses were performed using STATISTICA, version 6.0 , commercially available software package (Statistica for Windows, Statsoft, Tulsa, Oklahoma).

\section{RESULTS}

\section{Patient Characteristics}

The median age of patients was 61 years (range 20-85 years) (Table 2). The majority of patients $(90.5 \%)$ had a single lesion. The most frequently involved site was the temporal lobe $(23.6 \%$ among patients with a single lesion); in $32.2 \%$ of patients, more than one lobe was involved; in less than $2 \%$ of patients, 3 lobes were involved.

At the time of diagnosis, the presence of focal deficits was the most frequent symptom $(76.3 \%)$. Incidental diagnosis, based on 


\begin{tabular}{|c|c|}
\hline Feature & No. (\%) \\
\hline \multicolumn{2}{|l|}{ Age, y } \\
\hline$\leq 50$ & $215(20.3)$ \\
\hline $51-60$ & $312(29.5)$ \\
\hline$>60$ & $532(50.2)$ \\
\hline \multicolumn{2}{|l|}{ Sex } \\
\hline Male & $651(61.5)$ \\
\hline Female & $408(38.5)$ \\
\hline \multicolumn{2}{|l|}{ No. of lesions } \\
\hline Single & $958(90.5)$ \\
\hline Multiple & $101(9.5)$ \\
\hline Tumor site in patients with single lesion & $958(90.5)$ \\
\hline Supratentorial sites & $948(99.0)$ \\
\hline Cerebellum & $8(0.8)$ \\
\hline Brainstem & $2(0.2)$ \\
\hline \multicolumn{2}{|l|}{ Presenting symptoms } \\
\hline Focal symptoms & $808(76.3)$ \\
\hline Cranial hypertension & $336(31.7)$ \\
\hline Seizure & $215(20.3)$ \\
\hline None & $6(0.6)$ \\
\hline \multicolumn{2}{|l|}{ Preoperative imaging } \\
\hline MRI & $169(16.0)$ \\
\hline$C T$ & $226(21.3)$ \\
\hline$M R I+C T$ & $664(62.7)$ \\
\hline Functional imaging & $21(3.9)$ \\
\hline \multicolumn{2}{|l|}{ Postoperative imaging } \\
\hline$M R I$ & $206(19.4)$ \\
\hline$C T$ & $371(35.1)$ \\
\hline$M R I+C T$ & $376(35.5)$ \\
\hline None & $106(10.0)$ \\
\hline \multicolumn{2}{|l|}{ Preoperative KPS } \\
\hline $90-100$ & $288(27.2)$ \\
\hline 80 & $220(20.8)$ \\
\hline$\leq 70$ & $551(52.0)$ \\
\hline \multicolumn{2}{|l|}{ Preradiotherapy KPS } \\
\hline $90-100$ & $390(36.9)$ \\
\hline 80 & $334(31.5)$ \\
\hline$\leq 70$ & 335 (31.6) \\
\hline \multicolumn{2}{|l|}{ RPA class } \\
\hline III & $121(11.4)$ \\
\hline IV & $651(61.5)$ \\
\hline V & 197 (18.6) \\
\hline VI & $90(8.5)$ \\
\hline
\end{tabular}

${ }^{a} \mathrm{MRI}$, magnetic resonance imaging; $\mathrm{CT}$, computed tomography; KPS, Karnofsky Performance Status; RPA, recursive partitioning analysis. imaging performed without neurological symptoms, occurred in only $0.6 \%$ of cases.

Magnetic resonance imaging (MRI) was frequently used both in preoperatively and postoperatively $(78.7 \%$ and $54.9 \%$, respectively). Most patients $(\mathrm{n}=664,62.7 \%)$ underwent both preoperative MRI and computed tomography (CT), whereas only $35.5 \%$ of patients underwent both imaging studies postoperatively before RT.

The median preoperative and pre-RT Karnofsky Performance Status (KPS) scale scores were 70 and 80 , respectively. Most of the patients $(61.5 \%)$ met the criteria for recursive partitioning analysis (RPA) class IV (Table 3$).{ }^{4}$

\section{Treatment Characteristics}

\section{Surgery and Perioperative Therapy}

Biopsy was performed in only 123 patients (11.6\%), whereas most of the patients underwent craniotomy with removal of the lesion ( $\mathrm{n}=936,86.0 \%)$ (Table 4).

Postoperative imaging was performed in 953 patients (90.0\%), 901 of whom had undergone surgical removal. The scans in 710 of these patients ( $75.9 \%$ of cases of surgical removal) were adequate to assess the extent of surgery. For the remaining 226 patients (191 with difficult-to-interpret postoperative scans and 35 without postoperative scans), the extent of tumor eradication was based on surgical report alone ( $24.1 \%$ of patients who had undergone surgical tumor removal).

In $45.8 \%$ of the patients, surgery resulted in complete removal (no residual enhancement seen on postoperative CT or MRI or no residual tumor based on surgical reports when no postoperative imaging was available); partial removal occurred in $41 \%$. Carmustine wafers were used in 11 patients, and 2 patients underwent radioimmunotherapy in the adjuvant setting.

\section{Radiotherapy}

All 1059 patients received adjuvant RT. Approximately 90\% $(\mathrm{n}=934)$ of the patients were treated with 3-dimensional conformal RT (3D-CRT). A total of 163 treatment plans (17.4\% of patients treated with 3D-CRT) were based on coregistered CT and MRI. The vast majority of patients $(\mathrm{n}=903,85.3 \%)$ were treated with conventional fractionation ( 1.8 or 2 Gy per fraction, 5 fractions per week), with the median prescribed dose being 60 Gy (range 50-70 Gy). Among the patients treated with hypofractionation $(\mathrm{n}=156), 133$ patients received 3 Gy for daily fraction with a median total dose of $30 \mathrm{~Gy}$ (range 30-45 Gy).

\section{Adjuvant Chemotherapy}

A total of 287 patients $(27.1 \%)$ received RT alone, whereas 749 patients $(70.7 \%)$ underwent postoperative chemotherapy with TMZ. Concomitant and sequential TMZ was the most frequently adopted chemotherapy schedule $(\mathrm{n}=542,51.2 \%)$.

In 77 patients $(7.3 \%), 1$ cycle of TMZ $(200 \mathrm{mg} / \mathrm{mq})$ was administered before RT according to the design of an ongoing clinical trial. Sixty-four patients (6.0\%) received TMZ only during RT, 


\begin{tabular}{|c|c|c|c|c|c|c|c|}
\hline RPA Class & Age, y & Histotype & KPS & $\begin{array}{c}\text { Mental or } \\
\text { Neurological Status }\end{array}$ & Surgery & Symptom Onset & RT \\
\hline । & $<50$ & AA & - & Normal mental status & - & - & - \\
\hline II & $\geq 50$ & AA & $70-100$ & - & - & 3 mo before treatment & - \\
\hline \multirow[t]{2}{*}{ III } & $<50$ & AA & - & Abnormal mental status & - & - & - \\
\hline & $<50$ & GBM & $90-100$ & - & - & - & - \\
\hline \multirow[t]{3}{*}{ IV } & $<50$ & GBM & $<90$ & - & - & - & - \\
\hline & $\geq 50$ & AA & $70-100$ & - & - & $<3$ mo before treatment & - \\
\hline & $\geq 50$ & GBM & - & Good neurological function & Resection & - & - \\
\hline \multirow[t]{3}{*}{ V } & $\geq 50$ & GBM & $70-100$ & Disability to work & Resection & - & - \\
\hline & $\geq 50$ & GBM & $70-100$ & - & Biopsy & - & $\geq 54.4 \mathrm{~Gy}$ \\
\hline & $\geq 50$ & GBM & $<70$ & Normal mental status & - & - & - \\
\hline \multirow[t]{2}{*}{ VI } & $\geq 50$ & GBM & $70-100$ & - & Biopsy & - & $<54.4 \mathrm{~Gy}$ \\
\hline & $\geq 50$ & GBM or AA & $<70$ & Abnormal mental status & - & - & - \\
\hline
\end{tabular}

${ }^{a}$ RPA, recursive partitioning analysis; KPS, Karnofsky Performance Status; RT, radiotherapy; AA, anaplastic astrocytoma; GBM, glioblastoma multiforme.

whereas 66 patients $(6.2 \%)$ received only sequential TMZ after RT alone.

Only $2.2 \%$ of patients received different adjuvant chemotherapy (carmustine: $\mathrm{n}=9[0.8 \%]$; procarbazine + lomustine + vincristine: $\mathrm{n}=14[1.3 \%])$.

A total of 287 patients $(27.1 \%)$ did not receive chemotherapy. Among these patients, 192 (66.9\%) were elderly. The unsuitability of chemotherapy in 24 of the remaining 95 patients was based on poor prognostic factors that made the aim of treatment palliative. Seventy-one patients were treated exclusively with RT in centers where adjuvant chemotherapy with TMZ was proposed only after the publication of the phase III trial by Stupp et al. ${ }^{1}$

\section{Changes in Adjuvant Treatment Over Time}

Since the evidence-based data on efficacy of postoperative TMZ were disseminated in March 2005, patients were stratified according to the time of treatment (group A: from January 2002 to March 2005, the time of publication of phase III trial of the efficacy of TMZ in the adjuvant treatment; group B: from April 2005 to June 2007, the end of the current study). Use of chemotherapy significantly increased in the subgroup of patients treated from April $2005\left(\chi^{2}\right.$ test, $\left.P<.001\right)$. In the same group, the 3D-CRT was more often adopted compared with patients treated in the earlier years of the study $\left(\chi^{2}\right.$ test, $\left.P<.001\right)$ (Table 5).

\section{Age and Treatment}

Older patients (older than 60 years of age) were more often treated with a less aggressive approach. Surgical removal and 3DCRT were less frequently used in elderly patients, although differences did not reach statistical significance. At the same time, there was a significant difference in the choice of fractionation and systemic therapy across age groups. Hypofractionated treatment was given to a higher proportion of patients older than 60 years $(20.7 \%)$ compared with younger ones $\left(8.7 \% ; \chi^{2}\right.$ test, $\left.P<.001\right)$. Only $64 \%$ of patients older than the age of 60 years received adjuvant chemotherapy, whereas a larger percentage of younger patients were treated with systemic therapy $\left(82.0 \% ; \chi^{2}\right.$ test, $\left.P<.001\right)$.

\section{Salvage Treatment}

Salvage treatment was recorded for 709 patients. After progression, most of the patients $(\mathrm{n}=481,67.8 \%)$ were treated with chemotherapy (TMZ: $\mathrm{n}=203$, procarbazine + lomustine + vincristine: $\mathrm{n}=97$, fotemustine: $\mathrm{n}=78$, bleomycin and carmustine: $\mathrm{n}=54$, other: $\mathrm{n}=49)$. Forty-two patients $(5.9 \%)$ underwent $\mathrm{a}$ second surgery, whereas 10 patients $(1.4 \%)$ received radiosurgery or hypofractionated stereotactic RT as second RT. One hundred seventy-six patients $(24.8 \%)$ had best supportive care for the progressive disease.

\section{Survival}

For the entire series, the median survival was 9.5 months, and actuarial $O S$ rates at 1,2 , and 5 years were $62.3 \%, 24.8 \%$, and $3.9 \%$, respectively.

The median follow-up, calculated from the end of RT to last follow-up or death, was 6.5 months (follow-up was longer than 90 days after the end of RT in more than $85 \%$ of patients).

\section{Univariate Analysis}

Variables selected for survival analysis were chosen from both patient and treatment characteristics (Table 6).

Patient characteristics significantly associated with a better prognosis included younger age at diagnosis, single lesion, absence of focal symptoms at diagnosis, higher preoperative KPS scale score as well as pre-RT KPS scale score, and lower RPA class.

Treatment characteristics significantly associated with survival included more extensive surgery, use of 3D-CRT, 60 Gy as total 


\begin{tabular}{|c|c|}
\hline & No. (\%) \\
\hline \multicolumn{2}{|l|}{ Surgical procedure } \\
\hline Biopsy & $123(11.6)$ \\
\hline Craniectomy & $25(2.4)$ \\
\hline Craniotomy & $911(86.0)$ \\
\hline \multicolumn{2}{|l|}{ Extent of surgery } \\
\hline Gross total removal & $485(45.8)$ \\
\hline Subtotal removal & $434(41.0)$ \\
\hline Unknown & $17(1.6)$ \\
\hline Biopsy & $123(11.6)$ \\
\hline \multicolumn{2}{|l|}{ 3D-CRT } \\
\hline Yes & $934(88.2)$ \\
\hline No & $125(11.8)$ \\
\hline \multicolumn{2}{|l|}{ RT fraction size (Gy) } \\
\hline 1.8 & $45(4.3)$ \\
\hline 2 & $858(81.0)$ \\
\hline 2.5 & $23(2.2)$ \\
\hline 3 & $133(12.5)$ \\
\hline \multicolumn{2}{|c|}{ RT total dose (Gy) for $1.8 / 2$ Gy per fraction } \\
\hline Total & $903(85.3)$ \\
\hline$<59.4$ & $90(8.5)$ \\
\hline 59.4 or 60 & $619(58.4)$ \\
\hline$>60$ & $194(18.3)$ \\
\hline
\end{tabular}

Continues

radiation dose in conventionally fractionated treatments, and the use of chemotherapy (Figures 1-3).

Patients who received both concomitant $\left(75 \mathrm{mg} / \mathrm{m}^{2} /\right.$ day, 7 days per week) and sequential $\left(150 / 200 \mathrm{mg} / \mathrm{m}^{2}\right.$ for $5 \mathrm{~d}$ during each 28 -day cycle for 6 cycles) standard schedule of TMZ $(n=542)$ had a better survival compared with patients who were on different TMZ regimens $(n=207)$.

At the univariate analysis, treatment time after dissemination of the TMZ efficacy data was related to a better outcome (logrank test, $P=.03$ ).

We performed a univariate analysis in the subgroup of patients older than 60 years of age to evaluate how treatment modalities may

\begin{tabular}{|lc|}
\hline \multicolumn{2}{|l|}{ TABLE 4. (Continued) } \\
\hline
\end{tabular}

a 3D-CRT, 3-dimensional conformal radiotherapy; RT, radiotherapy; TMZ, temozolomide.

affect the survival of elderly patients. We found that aggressive treatment (surgical removal, 3D-CRT, conventionally fractionated treatment, and association with chemotherapy) was significantly associated with a better survival rate in the older age group (Table 7).

\section{Multivariate Analysis}

All the variables evaluated for the entire group were assessed using the Cox regression model. On multivariate analysis, 5 variables retained prognostic significance: age $(P=.002)$, pre-RT KPS scale score $(P=.01)$, extent of surgery $(P<.001)$, 3D-CRT $(P<$ $.001)$, and chemotherapy $(P<.001)$. Five variables among those that were significant in the univariate analysis failed to have a significant impact on survival: number of lesions; focal symptoms as presenting symptoms, preoperative KPS scale score, RPA class, and time of treatment (before or after dissemination of the TMZ efficacy data).

TABLE 5. Changes in Adjuvant Treatment Over Time ${ }^{a}$

\begin{tabular}{|c|c|c|c|c|c|c|c|}
\hline \multirow{2}{*}{ Time of Treatment } & \multirow{2}{*}{ Total No. } & \multicolumn{2}{|c|}{ Chemotherapy } & \multirow{2}{*}{$\chi^{2}$ Test } & \multicolumn{2}{|c|}{ 3D-CRT } & \multirow{2}{*}{$\chi^{2}$ Test } \\
\hline & & Yes & No & & Yes & No & \\
\hline January 2002-March 2005 & 435 & $268(61.6 \%)$ & $167(38.4 \%)$ & $P<.001$ & 365 (83.9\%) & $70(16.1 \%)$ & $P<.001$ \\
\hline
\end{tabular}

a 3D-CRT, 3-dimensional conformal radiotherapy. 


\begin{tabular}{|c|c|c|c|c|}
\hline & \multirow{2}{*}{$\begin{array}{c}\text { Log-Rank } \\
\text { Test } \\
P \text { Value }\end{array}$} & \multicolumn{3}{|c|}{ Proportion Surviving (\%) } \\
\hline & & 1 Year & 2 Years & 4 Years \\
\hline All & & 62.3 & 24.8 & 6.8 \\
\hline Age, y & $<.001$ & & & \\
\hline$\leq 50$ & & 75.4 & 39.5 & 16.4 \\
\hline $51-60$ & & 69.0 & 29.1 & 5.2 \\
\hline$>60$ & & 53.3 & 17.1 & 4.1 \\
\hline Sex & .14 & & & \\
\hline Male & & 61.4 & 22.5 & 5.3 \\
\hline Female & & 63.8 & 28.9 & 11.5 \\
\hline No. of lesions & $<.001$ & & & \\
\hline Single & & 63.6 & 26.7 & 7.3 \\
\hline Multiple & & 48.5 & 6.6 & NA \\
\hline \multicolumn{5}{|l|}{ Presenting symptoms } \\
\hline Cranial hypertension & .25 & & & \\
\hline Yes & & 57.4 & 22.2 & 6.8 \\
\hline No & & 64.6 & 26.2 & 6.8 \\
\hline Seizure & .16 & & & \\
\hline Yes & & 68.9 & 30.1 & 8.6 \\
\hline No & & 60.5 & 23.4 & 6.1 \\
\hline Focal symptoms & .01 & & & \\
\hline Yes & & 60.6 & 23.6 & 6.4 \\
\hline No & & 72.7 & 30.5 & 10.9 \\
\hline Preoperative KPS & $<.001$ & & & \\
\hline $90-100$ & & 70.1 & 29.6 & 9.7 \\
\hline 80 & & 66.0 & 25.8 & 6.6 \\
\hline$\leq 70$ & & 55.9 & 21.7 & 4.5 \\
\hline Preradiotherapy KPS & $<.001$ & & & \\
\hline $90-100$ & & 74.1 & 34.1 & 9.9 \\
\hline 80 & & 60.0 & 17.8 & 5.2 \\
\hline$\leq 70$ & & 48.8 & 21.6 & 5.3 \\
\hline RPA class & $<.001$ & & & \\
\hline III & & 80.6 & 46.5 & 18.8 \\
\hline IV & & 66.8 & 25.7 & 5.8 \\
\hline V & & 44.9 & 12.2 & 7.4 \\
\hline VI & & 40.2 & 17.8 & 0 \\
\hline Extent of surgery & $<.001$ & & & \\
\hline Biopsy & & 36.8 & 7.6 & NA \\
\hline Subtotal removal & & 58.8 & 22.7 & 8.5 \\
\hline Gross total removal & & 73.1 & 31.9 & 5.8 \\
\hline 3D-CRT & $<.001$ & & & \\
\hline Yes & & 64.4 & 26.8 & 7.4 \\
\hline No & & 41.8 & 4.7 & 0 \\
\hline
\end{tabular}

\begin{tabular}{|c|c|c|c|c|}
\hline & \multirow{2}{*}{$\begin{array}{c}\text { Log-Rank } \\
\text { Test } \\
P \text { Value }\end{array}$} & \multicolumn{3}{|c|}{ Proportion Surviving (\%) } \\
\hline & & 1 Year & 2 Years & 4 Years \\
\hline $\begin{array}{l}\text { RT total dose (Gy) for } \\
1.8 / 2 \text { Gy per fraction }\end{array}$ & $<.001$ & & & \\
\hline$<60$ & & 45.3 & 16.6 & NA \\
\hline 60 & & 70.7 & 32.9 & 8.5 \\
\hline$>60$ & & 64.3 & 21.3 & 6.5 \\
\hline $\begin{array}{l}\text { RT total dose (Gy) for } \\
3 \text { Gy per fraction }\end{array}$ & .15 & & & \\
\hline$\leq 30$ & & 20.2 & 5.4 & 0 \\
\hline$>30$ & & 41.9 & NA & NA \\
\hline Chemotherapy & $<.001$ & & & \\
\hline Yes & & 69.1 & 29.2 & 9.8 \\
\hline No & & 40.6 & 11.7 & 1.0 \\
\hline Temozolomide & $<.001$ & & & \\
\hline $\begin{array}{l}\text { Concomitant + } \\
\text { sequential TMZ }\end{array}$ & & 78.0 & 41.7 & 6.7 \\
\hline Other schedules of TMZ & & 62 & 20.3 & 10.0 \\
\hline Time of adjuvant treatment & .03 & & & \\
\hline January 2002-March 2005 & & 58.3 & 22.7 & 6.2 \\
\hline April 2005-June 2007 & & 65.8 & 26.7 & 7.7 \\
\hline
\end{tabular}

${ }^{a}$ NA, not available; KPS, Karnofsky Performance Status; RPA, recursive partitioning analysis; 3E-CRT, 3-dimensional conformal radiotherapy; TMZ, temozolomide.

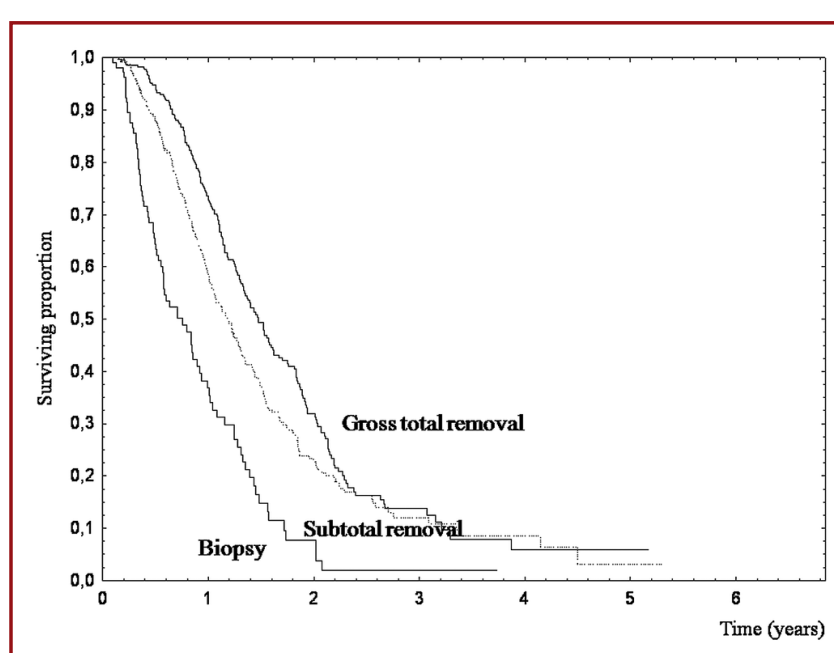

FIGURE 1. Extent of surgery and survival (log-rank test, $\mathrm{P}<.001$ ).

\section{Toxicity}

A retrospective assessment of toxicity was performed. Among patients who received RT alone $(\mathrm{n}=287), 26$ patients $(9.0 \%)$ had grade 2 headache, whereas grade 2 or higher worsening of preexisting focal neurological deficit was reported in 51 patients $(17.7 \%)$ (Table 8). 


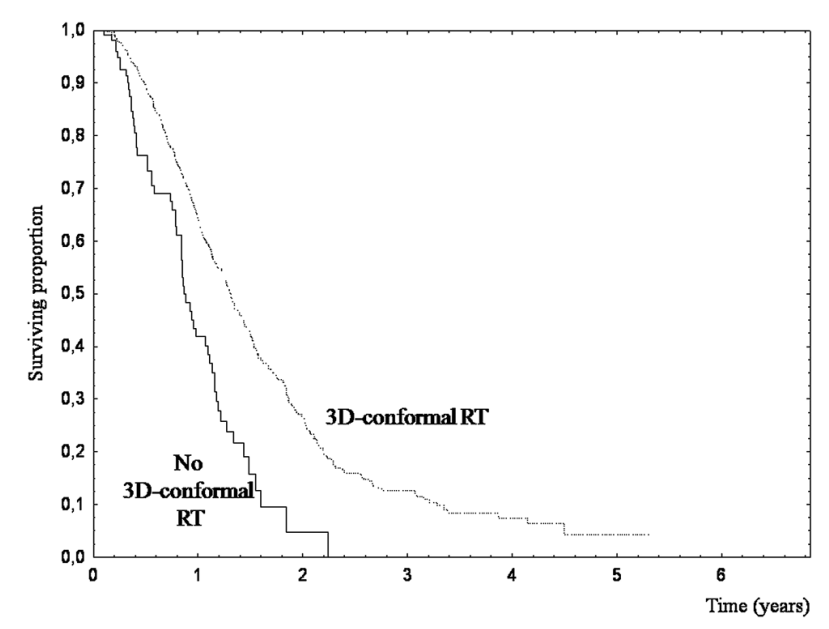

FIGURE 2. Three-dimensional conformal radiotherapy ( $3 D$-conformal $R T)$ and survival (log-rank test, $\mathrm{P}<.001$ ).

A total of 338 patients $(62.4 \%)$ did not show evidence of toxicity during concomitant RT and TMZ. During the concomitant phase, 361 toxic events were recorded. Severe myelodepression (grade 3 or higher) was recorded in 24 patients (4.4\%), and, consequently, all these patients prematurely discontinued TMZ treatment; in addition, 10 patients $(1.8 \%)$ received reduced doses of TMZ for the remaining weeks of treatment because of grade 2 hematological toxicity. In 6 patients $(1.1 \%)$ TMZ treatment was interrupted because of grade 3 deep venous thrombosis. There were 9 cases of grade 3 elevation of liver transaminases $(1.7 \%)$.

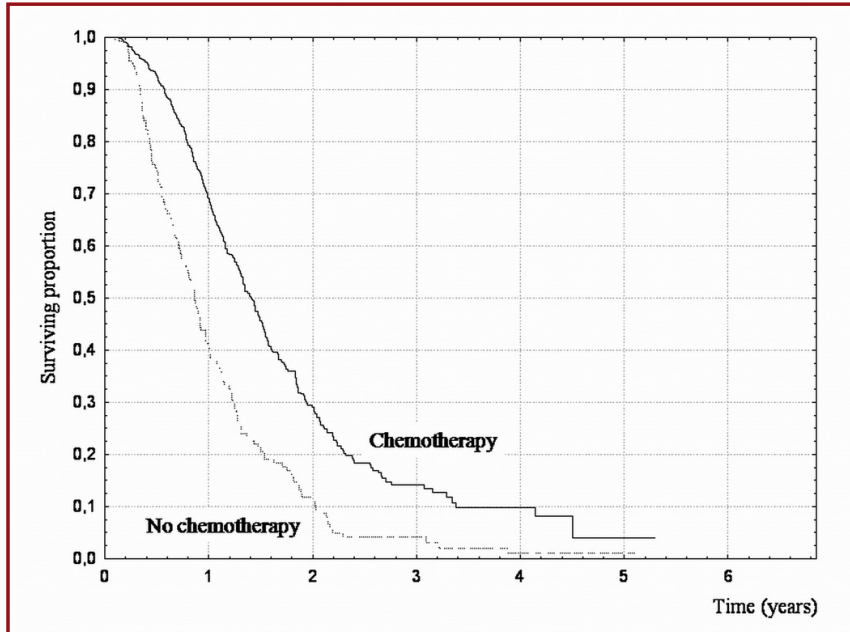

FIGURE 3. Postoperative chemotherapy and survival (log-rank test, $\mathrm{P}<.001$ ).

A total of 115 patients (21.3\%) had adverse effects during sequential TMZ treatment, for a total of 301 events. TMZ treatment was prematurely interrupted in 39 patients $(7.2 \%)$ because of grade 3 or higher hematological toxicity (neutropenia: $n=18$, thrombocytopenia: $\mathrm{n}=19$, anemia: $\mathrm{n}=2$ ), whereas 17 patients $(3.1 \%)$ required a dose reduction of TMZ for grade 2 myelodepression. Seven patients (1.3\%) discontinued TMZ because of grade 3 deep venous thrombosis. In addition, severe liver toxicity (grade 3 ) was reported in 5 cases $(0.9 \%)$.

The most common nonhematological adverse event during treatment was nausea, reported by $103(19.0 \%)$ and 107 (19.7\%) patients, during concomitant and sequential TMZ, respectively.

TABLE 7. Survival According to Treatment Modality in Elderly Patients

\begin{tabular}{|c|c|c|c|c|c|}
\hline \multirow{2}{*}{\multicolumn{2}{|c|}{ Age $>60 y, n=532(50.2 \%)$}} & \multirow{2}{*}{$\begin{array}{c}\begin{array}{c}\text { Log-Rank } \\
\text { Test } \boldsymbol{P} \text { Value }\end{array} \\
<.001\end{array}$} & \multicolumn{3}{|c|}{ Proportion Surviving (\%) } \\
\hline & & & 1 Year & 2 Years & 4 Years \\
\hline Exeresis & 465 (87.4\%) & & 56.1 & 19.2 & 4.8 \\
\hline \multicolumn{6}{|l|}{ RT } \\
\hline No & $72(13.5 \%)$ & & 40.9 & 0 & 0 \\
\hline Fractionation & & .004 & & & \\
\hline Conventional fractionated RT & $422(79.3 \%)$ & & 57.0 & 19.1 & 4.7 \\
\hline Hypofractionated RT & $110(20.7 \%)$ & & 34.1 & 5.7 & NA \\
\hline
\end{tabular}

a 3D-CRT, 3-dimensional conformal radiotherapy; RT, radiotherapy. 


\begin{tabular}{|c|c|c|c|c|}
\hline & G1 & G2 & G3 & G4 \\
\hline \multicolumn{5}{|l|}{$\begin{array}{l}\text { Toxicity for radiotherapy } \\
\text { alone }(n=287), \text { no. }(\%)\end{array}$} \\
\hline Headache & 40 (13.9) & $26(9.0)$ & - & - \\
\hline Nausea & $11(3.8)$ & - & - & - \\
\hline Emesis & $1(0.3)$ & - & - & - \\
\hline Neurological symptoms & $10(3.5)$ & $48(16.7)$ & $3(1.0)$ & - \\
\hline \multicolumn{5}{|l|}{$\begin{array}{l}\text { Toxicity for concomitant } \\
\text { temozolomide and radio- } \\
\text { therapy }(n=542), \text { no. }(\%)\end{array}$} \\
\hline Headache & $42(7.7)$ & $14(2.6)$ & - & - \\
\hline Nausea & $95(17.5)$ & $8(1.5)$ & - & - \\
\hline Emesis & $41(7.6)$ & $7(1.3)$ & - & - \\
\hline $\begin{array}{l}\text { Increased liver } \\
\text { transaminases }\end{array}$ & $12(2.2)$ & - & $9(1.7)$ & - \\
\hline Neutropenia & $8(1.5)$ & $11(2.0)$ & $3(0.5)$ & $6(1.1)$ \\
\hline Thrombocytopenia & $23(4.2)$ & $11(2.0)$ & $7(1.3)$ & $5(1.0)$ \\
\hline Anemia & $9(1.7)$ & $1(0.2)$ & $3(0.5)$ & - \\
\hline Deep venous thrombosis & - & - & $6(1.1)$ & - \\
\hline Fatigue & $28(5.2)$ & $12(2.2)$ & - & - \\
\hline \multicolumn{5}{|l|}{$\begin{array}{l}\text { Toxicity for sequential temo- } \\
\text { zolomide ( } n=542) \text {, no. }(\%)\end{array}$} \\
\hline Headache & $13(2.3)$ & $14(0.5)$ & - & - \\
\hline Nausea & $72(13.3)$ & $35(6.5)$ & - & - \\
\hline Emesis & $10(1.8)$ & $3(0.5)$ & - & - \\
\hline $\begin{array}{l}\text { Increased liver } \\
\text { transaminases }\end{array}$ & $17(3.1)$ & - & $5(0.9)$ & - \\
\hline Neutropenia & $12(2.2)$ & $12(2.2)$ & $9(1.7)$ & $9(1.7)$ \\
\hline Thrombocytopenia & $18(3.3)$ & $26(4.8)$ & $12(2.2)$ & $7(1.3)$ \\
\hline Anemia & $1(0.2)$ & $4(0.7)$ & $2(0.4)$ & \\
\hline Deep venous thrombosis & - & - & $7(1.3)$ & - \\
\hline Fatigue & $13(2.4)$ & - & - & - \\
\hline
\end{tabular}

${ }^{a} \mathrm{G}$, grade.

\section{Comparison With Previous Italian Patterns of Care Study}

In 2006, we reported the results of the Patterns of Care study based on the collaboration of 12 Italian RT centers that collected data over a 15-year period (1985-2001) on a total of 1722 glioma patients, the majority of whom were affected by glioblastoma $(\mathrm{n}=$ $1235,72 \%)^{3}$

In the current study, the results of a common database that included data on patients treated between 2002 and 2007 are reported. We compared data from the current study with those of the most recent period of the previous Italian study (1997-2001) to determine whether significant changes occurred in clinical practice during the past 10 years. The differences between these 2 periods of time were assessed with the $\chi^{2}$ test (Table 9). In both cases, most of the patients were preoperatively staged either with CT or MRI (61\% vs $62.7 \%, P=.48)$, but we found a significantly increased use of postoperative MRI ( $30 \%$ vs $54.9 \%, P<.001)$ either as the exclusive imaging modality $(14.0 \%$ vs $19.4 \%, P=.003)$ or as complementary to CT scanning ( $16 \%$ vs $35.5 \%, P<.001)$. The frequency of biopsies had decreased over time $(22 \%$ vs $11.6 \%, P<.001)$, with the majority of patients undergoing craniotomy. We did not find a significant change in the rate of gross total resection ( $48 \%$ vs $45.8 \%, P=.49)$, but in the current series, the extent of surgery was confirmed with postoperative MRI or CT most of the time (75.9\% of cases). The majority of patients included in the current series underwent $3 \mathrm{D}-\mathrm{CRT}$ ( $26 \%$ vs $88.2 \%$ ), suggesting the general acceptance of CT-based RT and, at the same time, an increased interest in treating glioblastoma patients with more complex techniques. Most of the patients $(85.1 \%)$ received a conventionally fractionated treatment, whereas there was a slight but significant increase in the proportion of patients receiving hypofractionated RT ( $10 \%$ vs $14.9 \%, P=.009)$. The role of chemotherapy in clinical practice has dramatically changed ( $21 \%$ vs $72.9 \%)$ since postoperative concurrent TMZ + RT followed by sequential TMZ had become the standard of care for newly diagnosed cases of glioblastoma during the accrual period.

Survival of the whole group of patients reported in the previous Italian study (1985-2001) significantly differs from the survival of the patients included in the current series (log-rank test, $P<.001)$ In comparing the survival of the subgroup of patients treated between 1997 and 2001 (median OS: 8.1 month, 1-year OS: $31.1 \%$, 2-year OS: 11.6\%) with the outcomes in the current study (median OS: 8.1 months, 1-year OS: 62.3\%, 2-year OS: $24.8 \%$ ), we found a significant improvement in terms of survival (log-rank test, $P<.001)$ (Figure 4 ).

\section{DISCUSSION}

\section{Comparison With Other Patterns of Care Studies}

This retrospective study examined patterns of care for newly diagnosed glioblastoma patients in Italy from 2002 to 2007. To our knowledge, it is the largest survey of glioblastoma patients for such a short period of observation. During this time period, the treatment for GBM significantly changed, mostly because RT + TMZ has become the standard of care. Thus, the analysis reflects an important period of change in the multidisciplinary management of glioblastoma.

In 2005, Chang et $\mathrm{al}^{5}$ reported the results of Glioma Outcomes Project (GOP), a multi-institutional database that monitored clinical practice patterns among North American patients with highgrade gliomas between 1997 and 2000. Stratification of patients according to sex, number of lesions, and seizure as a presenting sign produced results similar to ours (Table 10). The age-adjusted incidence was different in the 2 series, with the proportion of patients younger than 40 years being smaller in the current study $(9.8 \%$ vs $5.4 \%, P=.002)$. MRI was the most commonly performed imaging study in both series but was used more frequently in North America than in Italy $(P<.001)$. In the GOP study, 
TABLE 9. Main Differences With the Previous Italian Patterns of Care for Gliomas ${ }^{3 a}$

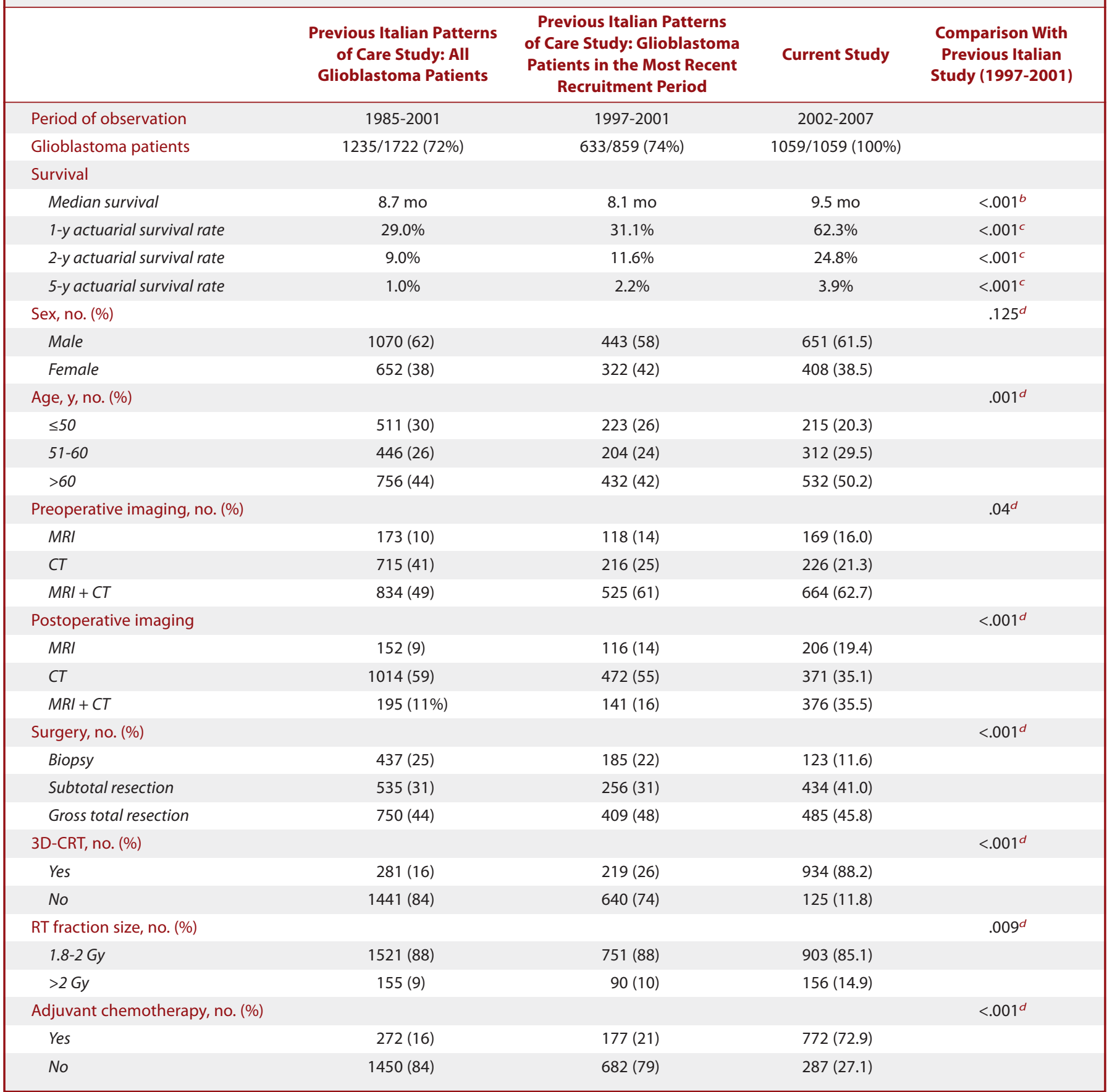

${ }^{a} \mathrm{MRI}$, magnetic resonance imaging; $\mathrm{CT}$, computed tomography; 3-CRT, 3-dimensional conformal radiotherapy; $\mathrm{RT}$, radiotherapy.

${ }^{b}$ F test, $P$ value.

${ }^{C}$ Log-rank test, $P$ value.

${ }^{d}$ Chi-square test, $P$ value.

biopsy was less frequently performed ( $4.6 \%$ vs $11.6 \%, P=.002)$. Data regarding the extent of tumor removal were not comparable because their study used surgical reports rather than postop- erative imaging. Intraoperative chemotherapy with carmustine wafers were used more frequently in the North American study than the current series. 


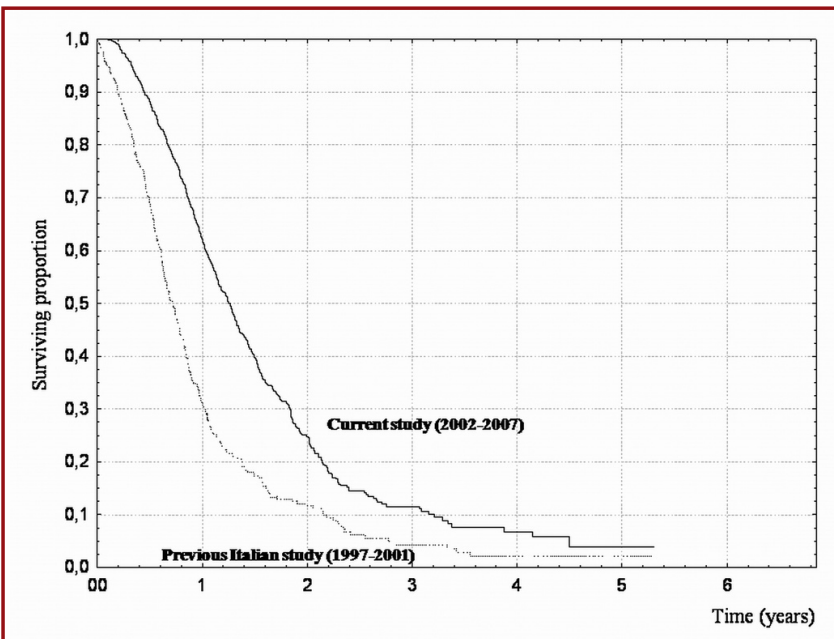

FIGURE 4. Survival of patients in the current series compared with the sur vival of patients recruited in the most recent accrual period of the previous Italian Patterns of Care study ${ }^{3}$ (log-rank test, $\left.\mathrm{P}<.001\right)$.

The proportion of patients receiving chemotherapy in the GOP survey was much lower than that of the present series. Furthermore, the authors reported that carmustine and lomustine were the most frequently used chemotherapy drugs since results of TMZ phase III trial ${ }^{1}$ were published some years after the end of accrual of the GOP survey.

Rosenthal et $\mathrm{al}^{6}$ recently reported an Australian Patterns of Care survey, examining data for $473 \mathrm{GBM}$ patients among 828 eligible patients with gliomas. In this study, the Victorian Cooperative Oncology Group examined patterns of clinical practice for patients with gliomas (World Health Organization I-IV) over a 3-year period (1998-2000) using a questionnaire developed by a multidisciplinary committee. Their questionnaire was designed to obtain data regarding patient characteristics and therapeutic management, but it did not evaluate number of lesions, presenting symptoms, KPS scale score, and diagnostic imaging procedures. Furthermore, details about RT (technique, total dose, fractionation) were not reported. Comparing their findings in GBM patients with ours, gross total removal was performed less frequently than in our series ( $31 \%$ vs $45.8 \%, \chi^{2}$ test, $\left.P<.001\right)$, although the authors reported the extent of tumor removal by surgical report alone. Only $86 \%$ of patients in the Australian study were referred to a RT center, and only 322 patients $(68 \%)$ underwent RT. Once again, the main difference compared with our results was the lower rate of adjuvant chemotherapy in the Victorian Patterns of Care $(P<.001)$. In a more recent series, $\mathrm{Cher}$ et $\mathrm{al}^{7}$ specified that the use of concurrent systemic therapy was extremely rare $(<1 \%)$, whereas $13 \%$ of cases performed post-RT chemotherapy.

\section{Survival and Prognostic Factors}

Median survival in the whole series was 9.5 months, similar to those of other published Patterns of Care studies (7.4-9.5 months). ${ }^{5,8}$ Two-year OS was higher than what was observed in other Patterns of Care studies (24.8\% vs 9\%-11\%., ${ }^{3,8}$ Survival has statistically improved compared with the previous Italian series ${ }^{3}$ and particularly the patients recruited in the last accrual period (1997-2001) (Figure 4).

Multivariate analysis showed a statistical significance for age, postoperative KPS scale score, extent of surgery, use of 3D-CRT, and use of chemotherapy.

Age was reported to be a significant prognostic factor in several Patterns of Care studies. ${ }^{3,8,9}$ In addition, postoperative performance data from the previous Italian survey ${ }^{3}$ and from the $\mathrm{GOP}^{5}$ were validated as having prognostic value. Extent of resection has also been shown to be an important factor in determining outcome in all the Patterns of Care studies that performed a survival analysis comparing subgroups of patients treated with different surgical modalities. ${ }^{3,9,10}$ Barnholtz-Sloan et $\mathrm{al}^{10}$ showed an impact on survival that depended on treatment modality, demonstrating an increased risk of death for patients treated with biopsy only, single-modality treatment (vs surgery $+\mathrm{RT}$ ), or no chemotherapy. Chemotherapy-treated patients also had a better outcome in the previous Italian study. ${ }^{3}$

All the parameters selected for multivariate analysis, except the use of 3D-CRT, were associated with a higher survival rate in the recently published analysis of the EORTC/NCIC trial 2698122981, aimed to derive nomograms for predicting survival of GBM patients. ${ }^{11}$

We observed a relatively long-term survival in subgroups of patients with the previously mentioned favorable prognostic factors: 4 -year OS ranging from $5.8 \%$ to $16.4 \%$ (Table 6).

\section{Elderly Patients: Prognosis and Treatment}

Advanced age, as previously mentioned, was associated with significantly lower survival in other Patterns of Care studies. ${ }^{3,8,9}$ Younger age has been validated as an independent prognostic factor also in the recently published subanalysis of the EORTC/NCIC trial 26981-22981. ${ }^{11}$ Our results confirm that older patients have a worse prognosis. Age proved to be a statistically significant prognostic factor in both univariate and multivariate analyses $(P<.001)$.

We found significant disparity in the adjuvant treatment prescribed across age groups. Patients older than 60 years were treated more often with hypofractionation and without chemotherapy compared with patients 60 years of and younger ( $P<.001$ for both).

Several Patterns of Care studies, some of which specifically focused on elderly patients, showed a significant difference in the use of postoperative therapy in the different age groups. $3,5,7,9,10,12$ Lowry et $\mathrm{al}^{9}$ reported that $18 \%$ of elderly patients (older than 65 years) diagnosed between 1990 and 1995 received no treatment at all, whereas $13 \%$ of cases underwent surgery alone. BarnholtzSloan et $\mathrm{al}^{10}{ }^{10}$ using the Surveillance, Epidemiology and End Results (SEER) database, identified 1753 cases of elderly GBM patients (older than 65 years) and concluded that age was strongly associated with treatment difference, even among older patients, with very elderly patients (older than 75 years) being more likely to receive limited treatment (surgery alone or RT alone). Lutterbach et $\mathrm{al}^{12}$ examined a large monoinstitutional cohort of glioblastoma 
TABLE 10. Main Differences With the Subgroup of Glioblastoma Patients Included in Glioma Outcome Project ${ }^{5,8 a}$

\begin{tabular}{|c|c|c|c|}
\hline & Glioma Outcome Project & Current Study & Statistical Comparison, $P$ Value \\
\hline Glioblastoma patients & $418 / 565(74 \%)$ & 1059/1059 (100\%) & \\
\hline Median survival & $9.5 \mathrm{mo}$ & $9.5 \mathrm{mo}$ & \\
\hline 2-y actuarial survival rate & $11.0 \%$ & $24.8 \%$ & \\
\hline Female & $162(39.6)$ & $408(38.5)$ & \\
\hline Age, y, no. (\%) & & & $.01^{b}$ \\
\hline$<40$ & $39(9.8)$ & $57(5.4)$ & \\
\hline $40-60$ & $165(41.5)$ & $470(44.4)$ & \\
\hline Multiple & $48(11.7)$ & $101(9.5)$ & \\
\hline Presenting symptoms and signs & & & $.22^{b}$ \\
\hline Seizure & $97(23.5)$ & $215(20.3)$ & \\
\hline Preoperative imaging & & & $<.001^{b}$ \\
\hline$M R I$ & $375(90.8)$ & $833(78.7)$ & \\
\hline$C T$ & $313(75.8)$ & $890(84.0)$ & \\
\hline Surgery, no. (\%) & & & $<.001^{b}$ \\
\hline Biopsy & $19(4.6)$ & $123(11.6)$ & \\
\hline Subtotal resection & $113(27.4)$ & $434(41.0)$ & \\
\hline Gross total resection & $192(46.5)$ & $485(45.8)$ & \\
\hline Postoperative RT, no. (\%) & & & $<.001^{b}$ \\
\hline Yes & $365(89.7)$ & $1059(100)$ & \\
\hline No & $53(10.3)$ & $0(0)$ & \\
\hline Adjuvant chemotherapy, no. (\%) & & & $<.001^{b}$ \\
\hline Yes & $222(54.6)$ & 772 (72.9) & \\
\hline No & $196(45.4)$ & $287(27.1)$ & \\
\hline
\end{tabular}

a Percentages from Glioma Outcome Project are based on the number of patients who answered each question on the questionnaire, as reported by the authors. Statistical comparison of outcome in terms of survival is not feasible because no measure of variability (no standard errors or standard deviations) was reported in the Glioma Outcome Project (thus making it impossible to apply statistical tests). NA, no available; MRI, magnetic resonance imaging; $\mathrm{CT}$, computed tomography; $\mathrm{BCNU}$, bleomycin and carmustine; KPS, Karnofsky Performance Status; RT, radiotherapy.

${ }^{b}$ Chi-square test, $P$ value.

patients $(n=430)$ and found more frequent use of 3D-CRT in younger patients. The previous Italian study ${ }^{3}$ already reported more frequent use of conventionally fractionated RT and chemotherapy in patients aged 60 and younger. Results of the $\mathrm{GOP}^{5}$ confirmed that patients receiving chemotherapy tended to be younger. Cher et $\mathrm{al}^{7}$ analyzed the use of chemotherapy in patients who were included in the Victorian Patterns of Care and found that only $15 \%$ of patients older than 60 years received chemotherapy. 
Our series showed that elderly patients undergoing aggressive treatment had a longer survival (Table 7), which was in accordance with other studies. ${ }^{913-15}$ These findings suggest that old age in itself should not preclude aggressive treatment when feasible.

\section{Toxicity of Adjuvant Treatment}

Despite the well-known limits of retrospective toxicity assessment, data regarding treatment-related side effects were collected. All adverse events were recorded and scored according to validated toxicity criteria. RT alone was globally well tolerated. Grade 3 central nervous system toxicity (neurological findings requiring hospitalization) was observed in only 3 patients $(1.0 \%)$. During concomitant RT + TMZ, most adverse events $(\mathrm{n}=322)$ were mild or moderate (grade 2 or lower), whereas severe toxicity was reported in 39 of 542 patients (7.2\%). Fifty-one patients (9.4\%) experienced grade 3 or 4 toxicity during sequential chemotherapy. The toxicity profile of RT + TMZ reported in the current retrospective series (and consequently limited by its own nature) was similar to that described in the EORTC/NCIC trial ${ }^{1}$ in which grade 3 or 4 hematological effects developed in $7 \%$ to $14 \%$ of patients during concomitant and sequential therapy, respectively.

\section{Limits and Advantages of the Study}

Examining 1059 biopsy-proven pure glioblastoma cases, this series differs from others that included different histological grades $^{3,5,6}$ or mixed gliomas with an oligodendroglial component ${ }^{5}$ as well as patients without histological diagnosis. ${ }^{6}$ Details on imaging, surgery (with emphasis on postoperative radiological assessment of surgical extent), RT, and chemotherapy make this analysis comprehensive. Moreover, it is the first survey that includes stratification of patients according to RPA classes and analysis of treatment-related toxicity scored according to validated criteria (while recognizing the limitations of retrospectively assessing toxicity).

The results, although limited by the retrospective nature of the study, may highlight that greater diagnostic and therapeutic resources are now offered to glioblastoma patients. More frequent use of postoperative MRI, for instance, might reflect greater attention to assessing the extent of surgical resection (and its "true" prognostic value) or to better defining RT target volume (using MRI for coregistration in the treatment planning phase). We think that the wide diffusion of postoperative MRI may also reflect the need to have a baseline radiological study for the follow-up, especially for a more accurate assessment of the early response to the treatment (ie, pseudoprogression features).

Even though some of the prognostic factors such as age, KPS scale score, and extent of surgery have been previously reported in literature, this is the first time that the use of 3D-CRT and the role of concomitant and sequential TMZ has been validated in such a large group of patients.

This study may be criticized for several limitations. First, the data are limited by the retrospective nature of the study with a high number of censored patients. Second, it included only patients who received RT, excluding patients who had a biopsy- proven glioblastoma but were not referred for RT. Third, the lack of biological data, such as $\mathrm{O}(6)$-methylguanine-DNA methyltransferase status, is a consistent limitation of our survey, considering the predictive and prognostic importance of such data. ${ }^{11,16}$ Moreover, the lack of a uniform pathological review remains a drawback of this study. Finally, we did not assess the quality of life of patients with validated tests nor we did collect data on supportive care (eg, steroids, antiepileptic drugs, thromboembolic prophylaxis).

\section{CONCLUSION}

This present study provides a benchmark analysis of current patterns of clinical practice and outcomes for patients with glioblastoma. Because of the time period covered (2002-2007), it revealed major changes in GBM patient care, highlighting the greater diagnostic and therapeutic resources that are now available to glioblastoma patients.

The routine use of MRI, the decision to perform a biopsy in only a minority of patients, the more frequent use of 3D-CRT, and the widespread use of chemotherapy represent the most significant changes in clinical practice reported in our analysis. The observed improvement in survival, with a significant proportion of relatively long-term survivors (4-year OS: 6.8\%; 5-year OS: $3.9 \%)$, suggests that most glioblastoma patients would benefit from aggressive multimodality therapy.

\section{Disclosure}

The authors have no personal financial or institutional interest in any of the drugs, materials, or devices described in this article.

\section{REFERENCES}

1. Stupp R, Mason WP, van den Bent MJ, et al. European Organisation for Research and Treatment of Cancer Brain Tumor and Radiotherapy Groups; National Cancer Institute of Canada Clinical Trials Group. Radiotherapy plus concomitant and adjuvant temozolomide for glioblastoma. $N$ Engl J Med. 2005;52(10):987-996.

2. Stupp R, Hegi ME, Mason WP, et al. European Organisation for Research and Treatment of Cancer Brain Tumour and Radiation Oncology Groups; National Cancer Institute of Canada Clinical Trials Group. Effects of radiotherapy with concomitant and adjuvant temozolomide versus radiotherapy alone on survival in glioblastoma in a randomised phase III study: 5 year analysis of the EORTC-NCIC trial. Lancet Oncol. 2009;10(5):459-466.

3. Magrini S, Ricardi U, Santoni R, et al. Patterns of practice and survival in a retrospective analysis of 1722 adult astrocytoma patients treated between 1985 and 2001 in 12 Italian radiation oncology centers. Int J Radiat Oncol Biol Phys. 2006;65(3):788-799.

4. Curran WJ Jr, Scott CB, Horton J, et al. Recursive partitioning analysis of prognostic factors in three Radiation Therapy Oncology Group malignant glioma trials. J Natl Cancer Inst. 1993;85(9):704-710.

5. Chang SM, Parney IF, Huang W, et al. Patterns of care for adults with newly diagnosed malignant glioma. JAMA. 2005;293(5):557-564.

6. Rosenthal MA, Drummond KJ, Dally M, et al. Management of glioma in Victoria (1998-2000): retrospective cohort study. Med J Aust. 2006;184(6):270-273.

7. Cher L, Rosenthal MA, Drummond KJ, et al. The use of chemotherapy in patients with gliomas: patterns of care in Victoria from 1998-2000. J Clin Neurosci. 2008;15(4):398-401.

8. Laws ER, Parney IF, Huang W, et al. Survival following surgery and prognostic factors for recently diagnosed malignant glioma: data from the Glioma Outcomes Project. J Neurosurgery. 2003;99(3):467-473. 
9. Lowry JK, Snyder JJ, Lowry PW. Brain tumors in the elderly: recent trends in a Minnesota cohort study. Arch Neurol. 1998;55(7):922-928.

10. Barnholtz-Sloan JS, Williams VL, Maldonado JL, et al. Patterns of care and outcomes among elderly individuals with primary malignant astrocytoma. J Neurosurg. 2008;108(4):642-648.

11. Gorlia T, van den Bent MJ, Hegi ME, et al. Nomograms for predicting survival of patients with newly diagnosed glioblastoma: prognostic factor analysis of EORTC and NCIC trial 26981-22981/CE.3. Lancet Oncol. 2008;9(1):29-38.

12. Lutterbach J, Bartelt S, Momm F, Becker G, Frommhold H, Ostertag C. Is older age associated with a worse prognosis due to different patterns of care? A longterm study of 1346 patients with glioblastomas or brain metastases. Cancer. 2005;103(6):1234-1244.

13. Brandes AA, Vastola F, Basso U, et al. A prospective study on glioblastoma in the elderly. Cancer. 2008;97(3):657-662.

14. Mukerji N, Rodrigues D, Hendry G, Dunlop PR, Warburton F, Kane PJ. Treating high grade gliomas in the elderly: the end of ageism? J Neurooncol. 2008;86(3):329336.

15. Shaw EG. Nothing ventured, nothing gained: treatment of glioblastoma multiforme in the elderly. J Clin Oncol. 2004;22(9):1540-1541.
16. Hegi ME, Diserens AC, Gorlia T, et al. MGMT gene silencing and benefit from temozolomide in glioblastoma. $N$ Engl J Med. 2005;352(10):997-1003.

\section{Acknowledgments}

We thank the following colleagues for their valuable collaboration in collecting data: Barbara Grilli Leonulli, MD, (Florence), Icro Meattini, MD, (Florence), Paola Vitali, MD, (Brescia), Elena Montani, MD, (Brescia), Barbara Lazzari, MD, (Brescia), Angela Botticella, MD, (Torino), Stefano Dall'Oglio, MD, (Verona), Laura Masini, MD, (Novara), Annalisa Natuno, MD, (San Giovanni Rotondo), Patrizia Giacobazzi, MD, (Modena), Adele Piscopo, MD, (L'Aquila), Antonio Pontoriero, MD, (Messina), Carlo Ivan Cusano, MD, (Benevento), Fabrizio Matteucci, MD, (Pisa), Viviana Barbieri, MD, (Rionero in Vulture), Roberto Manzo, MD, (Naples), Luana Di Murro, MD, (Rome), Rebeca Nicolais, MD, (Rome), Ida Milanesi, MD, (Milan), Alessia Farneti, MD, (Perugia). S.S. thanks Alberto Iannalfi, MD, for the helpful support during the process of building the computerized database. We are also very grateful to David Biro, MD, who reviewed the English version of the manuscript; his help was invaluable.

\section{INTERNATIONAL TRAVELING FELLOWSHIP IN PEDIATRIC NEUROSURGERY}

The Joint Pediatric Neurosurgery Section of the American Association of Neurological Surgeons and Congress of Neurological Surgeons has established an international traveling fellowship for neurosurgeons who at the time of their application are either training in a residency program outside the United States and Canada, or who have completed residency training outside the United States and Canada within the past five years.

The fellowship will cover the traveling and living expenses for a three-month period to be spent observing the activities of an established Pediatric Neurosurgical service of the applicant's choosing in the United States or Canada. One fellowship will be awarded yearly on the basis of a competitive evaluation by a committee of the Pediatric Section at the annual meeting in December. The maximum fellowship stipend is $\$ 5000$.

The application must include:

1) A statement defining the purpose of the proposed fellowship;

2) A letter of recommendation from the applicant's current Neurosurgical program director;

3) A letter of acceptance from the institution where the applicant intends to take the fellowship, confirming the description of the fellow's potential activities during the period of the award;

4) The applicant's current Curriculum Vitae.

The strict deadline for application submission is November 15, 2010

The completed application should be sent to:

Bermans J. Iskandar, M.D.

Department of Neurological Surgery

University of Wisconsin School of Medicine and Public Health

600 Highland Avenue, Room K4/832 CSC

Madison, WI 53792

[or via e-mail to: iskandar@neurosurg.wisc.edu] 